\title{
Frame Design for 5G Multicarrier Modulations
}

\author{
S. Morosi*, M. Biagini*, F. Argenti*, E. Del Re*, and L. Yessenturayeva ${ }^{\dagger}$ \\ ${ }^{*}$ CNIT - DINFO, University of Florence, via S. Marta 3, 50139 Florence, Italy \\ Email: simone.morosi@unifi.it, massimiliano.biagini@unifi.it \\ ${ }^{\dagger}$ Kazakh National Technical University after K.I. Satpaev, Almaty, Kazachstan
}

\begin{abstract}
Orthogonal Frequency Division Multiplexing (OFDM) transmission technique has been considered for the 4G LTE-Advanced system because of its high performance and low complexity. In the new 5G scenario this type of modulation is not properly suited because of the additional requirements for very high spectral efficiency, low latency and low out-of-band (OOB) emissions. Thus, new waveforms have been chosen to replace it and effectively deal with these problems. Particularly, recently proposed multicarrier techniques as the Generalized Frequency Division Multiplexing (GFDM) and the Filter Bank Multicarrier (FBMC) modulations are two of the main candidates techniques for the new 5G standard. As for classical OFDM technique, the channel estimation is a crucial functionality for the all multicarrier approaches. In this paper we generalize a recently proposed method for the computation of the MSE on the time-frequency domain for pilot based channel estimation techniques in multicarrier system. This method permits to determine the most suitable channel estimation algorithm for a given wireless channel as well as to define the suitable pilot density in order to afford a target MSE value for a given SNR level. Hence, in this paper a general design tool for multicarrier systems is proposed.
\end{abstract}

\section{INTRODUCTION}

The Orthogonal Frequency Division Multiplexing (OFDM) transmission technique has been identified as the most appropriate solution in order to cope with the ever increasing requirements which will have to be afforded by the wireless communication systems as LTE-Avanced, for what concerns the data-rate as well as the Quality of Service (QoS) and the spectral efficiency [1]-[3]. As it is well known, the advantages of OFDM include implementation simplicity, capability to reduce multipath channel effects and the possibility to implement time-frequency channel dependent scheduling. However, in the next 5G scenario the OFDM presents some impairements with respect to the envisaged service requirements. Particularly, in the machine-to-machine (M2M) communication scenario, low power consumption is required, which is facilitated by a very strict synchronization in order to mantain the ortogonality between subcarriers. Furthermore, since OFDM presents a low spectral efficiency due to the insertion of ciclix prefix in each OFDM symbol, it is not suitable neither for tactile internet scenario nor in the vehicle-to-vehicle $(\mathrm{V} 2 \mathrm{~V})$ communication as well as in the wireless regional area networks (WRAN) applications. Additionally, the high OOB emissions of OFDM raises problems for dynamic spectrum access as well as for the exploitation of the white space [4] [5].

As a result, in the $5 \mathrm{G}$ context new waveforms are foreseen in order to cope with these problems: in particular Generalized Frequency Division Multiplexing (GFDM) [6] [7] and Filter Bank Multicarrier (FBMC) modulations [8] [9] are two of the main candidates techniques for the upcoming $5 \mathrm{G}$ standard.

In the GFDM each subcarrier is filtered with a nonorthogonal prototype filter, so reducing the OOB emmissions and permitting dynamic spectrum allocation and a higher spectral effiviency as well as a lower latency. The subcarrier filtering result in non-orthogonal subcarriers and both intersymbol interference (ISI) and inter-carrier interference (ICI) arise. Nevertheless, this interference can be eliminated by an efficient receiving technique, e.g., a matched filter receiver with iterative interference cancellation.

On the other hand, the FBMC does not require the CP insertion, so increasing the spectral efficiency; besides, instead of using a rectangular filter as in OFDM, the FBMC resorts to a more advanced pulse shaping which permits to model the spectrum emissions and reducing the OOB, as in the GFDM. The use of the FBMC with appropriate filter prototypes allows to obtain an almost negligible inter-symbol interference (ISI) and inter-carrier interference (ICI) assuming that the channel coherence bandwidth is enough large.

As for classical OFDM technique, the channel estimation is a crucial functionality for the all multicarrier approaches: also for the newly proposed systems, reliable channel estimators are required at the receiver side in order to perform an effective channel equalization [10] at the receiver side. Therefore, the channel estimation keeps on playing a fundamental role in the equalization procedure and in the determination of the receiver performance [2], [11]-[13]. Moreover, the frequency selectivity and the time-varying nature of the channels can cause a strong reduction of the performance of these wireless multicarrier communications.

As for the estimation techniques, pilot based approaches can be adopted for their reduced computational burden and remarkable performance: overviews of some of the pilot aided channel estimation techniques can be found in [14] and [15].

An evaluation tool that allows the determination of the channel estimation MSE for the aforementioned multicarrier signals ends up being very important in order to determine the performance of the estimators and the best position of the pilots in the frame. It is important to note that the tools which have been proposed for OFDM so far allow the MSE to be computed only on pilot-carrying symbols.

In this paper we generalize a recently proposed method [16]-[18] for the computation of the MSE on the time- 
frequency domain for pilot based channel estimation techniques in multicarrier system. This method permits to determine the most suitable channel estimation algorithm for a given wireless channel and the most appropriate pilot symbols pattern for any multicarriers transmission format.

Furthermore,the proposed approach permits to define the suitable pilot density in order to afford a target MSE value for a given SNR level. Therefore, in this paper a general design tool for multicarrier systems is defined.

Notations: In the following, matrix variables are indicated with boldface uppercase letters; vector variables with boldface lowercase letters, boldface uppercase calligraphic letters and boldface uppercase letters with a $v$ superscript; $\operatorname{tr}\{\mathbf{A}\}$ denotes the trace of the square matrix $\mathbf{A} ; \mathbf{A}^{v}=\operatorname{vec}(\mathbf{A})$ denotes a vector obtained stacking the columns of the matrix $\mathbf{A}$; the symbol $\otimes$ denotes the Kroneker's product between two matrices; $|\mathcal{S}|$ denotes the cardinality of the set $\mathcal{S} ; \mathbf{I}_{M}$ denotes the $M \times M$ identity matrix;

\section{SYSTEM MODEL}

Considering multicarrier systems such as OFDM, GFDM and FBMC [6], [8], [14], the following general form can be used in order to describe the transmitted signal

$$
x(n)=\sum_{m=0}^{M-1} \sum_{k=0}^{K-1} a_{k, m} g_{T}(n-m N) e^{\frac{j 2 \pi k n}{N}} n=0 \ldots N
$$

Particularly, in the FBMC modulation scheme, the transmitted signal is the sum of the outputs of a bank of $K$ filters. With respect to the OFDM modulation there are two main differences, namely the trasmitted prototype filter $g_{T}$ and the lack of the Cyclic Prefix (CP). In particular the OFDM technique adopts a rectangular filter, whereas in the FBMC approach a non-rectangular shaping filter is considered as in [8] [9].

On the other hand, with respect to both the OFDM and the FBMC where only $K$ symbols are processed, in GFDM technique the data source provides a binary data vector block of $K M$ symbols that are processed simultaneously. Thus, in this case $a_{k, m}$ is the symbol associated to the $k$-th subcarrier at the $m$-th timeslot [6] [7]. Moreover, in the GFDM each $a_{k, m}$ is transmitted with the corresponding non-rectangular shaping filter as in the following [6]

$$
g_{T_{k, m}}[N]=g[(n-m K) \bmod N] e^{\frac{-j 2 \pi k n}{K}}
$$

Therefore, each $g_{T_{k, m}}[n]$ is a time and frequency shifted version of a prototype filter $g_{T}$, where the modulo operation makes $g_{T_{k, m}}[n]$ a circularly shifted version of $g_{T_{k, 0}}[n]$, and the complex exponential performs the shifting operation in frequency [6] [7]. In all of these multicarriers modulation techniques, as shown in [14] [19] [20], the relation between the tranmitted and the received signals can be expressed as

$$
Y[k, m]=X[k, m] H[k, m]+W[k, m]
$$

where $Y[k, m]$ and $X[k, m]$ are the received and the transmitted signals, respectively, $k$ is the index over the frequency carriers $(k=0,1, \ldots, K-1), m$ is the index over the time symbols ( $m=0,1, \ldots, M-1)$, the term $W$ takes into account the AWGN noise with zero mean and variance $\sigma_{w}^{2}$, and $H$ represents the channel frequency response. It is important to stress that in both the GFDM and the FBMC approaches, both ICI and ISI will be experienced due to the lack of ortogonality which is introduced by the non-rectanguler filter. In [19] and [20] suitable techniques are presented which allow to cancel interference.

Thanks to the relation expressed in II, pilot-aided channel estimation techniques can be applied in these three communication systems and a general performance analysis can be derived. Therefore, in the following we denote with $\mathbf{X}_{p}=$ $\operatorname{diag}\left(x_{1}, x_{2}, \ldots \ldots, x_{p}\right)$, the $P \times P$ matrix of transmitted pilot symbols, with $\mathcal{H}_{p}$ the channel frequency response at the pilot locations, and with $\mathcal{Y}_{p}$ the $P \times 1$ received signal vector at the pilot positions, respectively. The channel frequency response estimation over the pilot carriers can be achieved by multiplying $\mathcal{Y}_{p}$ for the matrix $\mathbf{X}_{p}^{H}$ as in II

$$
\hat{\mathcal{H}}_{p}=\mathbf{X}_{p}^{H} \mathcal{Y}_{p}=\mathcal{H}_{p}+\mathbf{X}_{p}^{H} \mathcal{W}_{p}
$$

where $\hat{\mathcal{H}}_{p}$ represents the $P$-component vector of the estimated channel coefficients over pilot subcarriers. Pilot-aided channel estimation algorithms exploit this result to obtain an estimation on all the other subcarriers through interpolation and estimation methods. In the next section we will derive the mathematical model which refers to the pilotaided channel estimation techniques and can be applied to the three multicarrier communication systems which have been presented previously: this approach generalizes a recently proposed method [16]-[18] for the computation of the MSE on the time-frequency domain for pilot based channel estimation techniques.

\section{MATHEMATICAL MODEL}

Since the interpolation in the frequency domain is a linear operator and thanks to the knowledge of the vectors $\hat{\mathcal{H}}_{p}$, we can express the interpolated pilot coefficient values as

$$
\hat{\mathcal{H}}_{f}=\mathbf{A}_{f} \hat{\mathcal{H}}_{p}
$$

where $\mathbf{A}_{f}$ represents a $K \times P$ matrix containing the interpolation coefficients derived from the applied interpolation method and will not be presented for sake of conciseness. Afterwards, let $\hat{\mathbf{H}}_{f}$ be the matrix whose columns are the frequency-interpolated vectors $\hat{\mathcal{H}}_{f}$, and $\mathcal{M}$ be the set of time indexes of the pilot-carrying symbols within a frame, then the matrix $\hat{\mathbf{H}}_{f}$ has dimension $K \times|\mathcal{M}|$ and can be expressed by

$$
\hat{\mathbf{H}}_{f}=\left[\hat{\mathcal{H}}_{f}\left[i_{0}\right] \hat{\mathcal{H}}_{f}\left[i_{1}\right] \ldots \hat{\mathcal{H}}_{f}\left[i_{|\mathcal{M}|}\right]\right]
$$

where the index $0 \leq i_{k} \leq|\mathcal{M}|-1$ denotes the position of the pilot-carrying symbols within the frame.

Now, let $\hat{\mathbf{H}}_{p}$ be the $P \times|\mathcal{M}|$ matrix containing the estimated channel coefficients for the pilot symbols, that is

$$
\hat{\mathbf{H}}_{p}=\left[\hat{\mathcal{H}}_{p}\left[i_{0}\right] \hat{\mathcal{H}}_{p}\left[i_{1}\right] \ldots \hat{\mathcal{H}}_{p}\left[i_{|\mathcal{M}|}\right]\right]
$$


Making use of (5), a convenient form for expressing the content of $\hat{\mathbf{H}}_{f}$ is given by

$$
\hat{\mathbf{H}}_{f}^{v}=\left(\begin{array}{cccc}
\mathbf{A}_{f, i_{0}} & \mathbf{0} & \ldots & \mathbf{0} \\
\mathbf{0} & \mathbf{A}_{f, i_{1}} & \ldots & \mathbf{0} \\
\vdots & \vdots & \ddots & \vdots \\
\mathbf{0} & \mathbf{0} & \ldots & \mathbf{A}_{f, i_{|\mathcal{M}|}}
\end{array}\right) \hat{\mathbf{H}}_{p}^{v}=\mathbf{A}_{f d} \hat{\mathbf{H}}_{p}^{v}
$$

where $\mathbf{A}_{f d}$ is a block diagonal matrix containing all the interpolation coefficients of matrix $\mathbf{A}_{f}$ for the pilot-carrying OFDM symbols. Consider now the application of the timeinterpolation to $\hat{\mathbf{H}}_{f}$ and let $\hat{\mathbf{H}}$ be the complete estimated channel coefficients matrix, having dimension $K \times M: \hat{\mathbf{H}}$ is obtained by applying an interpolation operator to the rows (time direction) of $\hat{\mathbf{H}}_{f}$. Let $\mathbf{B}_{t}$ be the $M \times|\mathcal{M}|$ timeinterpolation operator. Since it operates on column vectors, we have

$$
\hat{\mathbf{H}}^{T}=\mathbf{B}_{t} \hat{\mathbf{H}}_{f}^{T}
$$

From (9) we have

$$
\operatorname{vec}\left(\hat{\mathbf{H}}^{T}\right)=\operatorname{vec}\left(\mathbf{B}_{t} \hat{\mathbf{H}}_{f}^{T}\right)
$$

Define the $m n \times m n$ permutation matrix $\mathbf{T}_{m, n}$ that transforms $\operatorname{vec}(\mathbf{X})$ into $\operatorname{vec}\left(\mathbf{X}^{T}\right)$, where $\mathbf{X}$ is a $m \times n$ matrix

$$
\operatorname{vec}\left(\mathbf{X}^{T}\right)=\mathbf{T}_{m, n} \operatorname{vec}(\mathbf{X})
$$

It can be shown that, if $\mathbf{Y}$ is a $p \times q$ matrix, then we have

$$
(\mathbf{X} \otimes \mathbf{Y}) \mathbf{T}_{n, q}=\mathbf{T}_{m, p}(\mathbf{Y} \otimes \mathbf{X})
$$

Consider also the following property of the $\operatorname{vec}(\cdot)$ operator:

$$
\operatorname{vec}(\mathbf{X Y W})=\left(\mathbf{W}^{T} \otimes \mathbf{X}\right) \operatorname{vec}(\mathbf{Y})
$$

Using (11)-(13) into (10) and identifying $\mathbf{W}=\mathbf{I}_{K}$ yields

$$
\begin{aligned}
& \mathbf{T}_{K, M} \operatorname{vec}(\hat{\mathbf{H}})=\left(\mathbf{I}_{K} \otimes \mathbf{B}_{t}\right) \operatorname{vec}\left(\hat{\mathbf{H}}_{f}^{T}\right) \\
& =\left(\mathbf{I}_{K} \otimes \mathbf{B}_{t}\right) \mathbf{T}_{K,|\mathcal{M}|} \operatorname{vec}\left(\hat{\mathbf{H}}_{f}\right) \\
& =\mathbf{T}_{K, M}\left(\mathbf{B}_{t} \otimes \mathbf{I}_{K}\right) \operatorname{vec}\left(\hat{\mathbf{H}}_{f}\right)
\end{aligned}
$$

Considering that $\mathbf{T}_{K, M}$ is invertible, we have

$$
\hat{\mathbf{H}}^{v}=\left(\mathbf{B}_{t} \otimes \mathbf{I}_{K}\right) \hat{\mathbf{H}}_{f}^{v}
$$

By using (8), we have

$$
\hat{\mathbf{H}}^{v}=\left(\mathbf{B}_{t} \otimes \mathbf{I}_{K}\right) \mathbf{A}_{f d} \hat{\mathbf{H}}_{p}^{v}=\mathbf{A}_{f, t} \hat{\mathbf{H}}_{p}^{v}
$$

Equation (16) expresses the interpolated channel coefficients, for the whole subframe, as a linear function of the estimated pilot coefficients. The other class of considered algorithm is based on the estimation of the channel impulse response by means of least square (LS), maximum likehood (ML) or minimum mean square error (MMSE) methods [14] [21]. Once the channel impulse response estimation $\hat{\mathbf{h}}$ is obtained, the DFT is applied as follow in order to obtain the vectro $\hat{\mathcal{H}}_{f}$ as following

$$
\hat{\mathcal{H}}_{f}=\mathbf{F}_{L} \hat{\mathbf{h}}
$$

At this point a time-interpolation according to the mathematical model presented is performed in order to complete the estimation on the whole frame. In this case the matrix $\hat{\mathbf{H}}_{f}$ has the following form

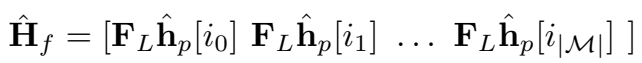

so that

$$
\hat{\mathbf{H}}_{f}^{v}=\mathbf{F}_{1} \hat{\mathbf{h}}_{p}^{v}
$$

where $\hat{\mathbf{h}}_{p}^{v}$ is a vector containing the time-domain channel impulse responses estimated over the pilot-carrying symbols, $\mathbf{F}_{L}$ is the Fourier transform matrix which represents the DFT of the channel impulse response, and $\mathbf{F}_{1}=\mathbf{I}_{|\mathcal{M}|} \otimes \mathbf{F}_{L}$. By applying the time-domain interpolation operator in (15), we achieve the interpolated channel coefficients for the whole subframe as

$$
\hat{\mathbf{H}}^{v}=\left(\mathbf{B}_{t} \otimes \mathbf{I}_{K}\right) \mathbf{F}_{1} \hat{\mathbf{h}}_{p}^{v}=\mathbf{B}_{1} \hat{\mathbf{h}}_{p}^{v}
$$

\section{MSE COMPUTATION}

The MSE analysis is a common way to measure the channel estimation performance, and to design the optimum pilot sequences and their position [13] [14] [22]. Let $\Theta$ be a $J$ vector of parameters (in our case, the true channel coefficients) and let $\hat{\boldsymbol{\Theta}}$ be an estimate of $\boldsymbol{\Theta}$. The MSE between the true and the estimated variables is defined as

$$
\begin{aligned}
\sigma_{I}^{2}= & \frac{1}{J} \mathrm{E}\left[\|\hat{\boldsymbol{\Theta}}-\boldsymbol{\Theta}\|_{2}^{2}\right]=\frac{1}{J}\left[\operatorname{tr}\left\{\mathrm{E}\left[\hat{\boldsymbol{\Theta}} \hat{\boldsymbol{\Theta}}^{H}\right]\right\}-\operatorname{tr}\left\{\mathrm{E}\left[\boldsymbol{\Theta} \hat{\boldsymbol{\Theta}}^{H}\right]\right\}\right. \\
& \left.-\operatorname{tr}\left\{\mathrm{E}\left[\hat{\boldsymbol{\Theta}} \boldsymbol{\Theta}^{H}\right]\right\}+\operatorname{tr}\left\{\mathrm{E}\left[\boldsymbol{\Theta} \Theta^{H}\right]\right\}\right]
\end{aligned}
$$

The expression in (21) is usually used to compute the MSE for the pilot-carrying symbols. The goal of this study is the derivation in a closed form of the MSE expression of a general multicarrier system in the time-frequency domain. Therefore, to this aim, the variables $\Theta$ and $\hat{\boldsymbol{\Theta}}$ has to contain the real channel and the estimated coefficients (exploiting the time domain interpolation) on the whole frame, respectively. Our computation method provides a general tool that can be applied to all linear channel estimation algorithms; moreover, it can be used as a support to the design process in order to obtain the best channel estimation algorithms, and to define the suitable pilot density in order to afford a target MSE value for a given SNR level. In the next two sections we will compute the MSE for the the whole multicarrier frame for the two classes of considered algorithms exploiting the previously introduced mathematical model.

\section{A. Time-Frequency MSE evaluation for channel estimation} algorithms based on separable interpolation

By using (16) and (21), and substituting $\boldsymbol{\Theta}=\mathbf{H}^{v}$ and $\hat{\boldsymbol{\Theta}}=$ $\mathbf{A}_{f, t} \hat{\mathbf{H}}_{p}^{v}$ we obtain the total MSE

$$
\begin{aligned}
\sigma_{I}^{2}= & \frac{1}{J}\left[\operatorname { t r } \left\{\mathbf{A}_{f, t} \mathrm{E}\left[\hat{\mathbf{H}}_{p}^{v}\left(\hat{\mathbf{H}}_{p}^{v}\right)^{H} \mathbf{A}_{f, t}^{H}\right]-\mathbf{A}_{f, t} \mathrm{E}\left[\mathbf{H}_{p}^{v}\left(\hat{\mathbf{H}}^{v}\right)^{H}\right]\right.\right. \\
& \left.\left.-\mathrm{E}\left[\mathbf{H}^{v}\left(\hat{\mathbf{H}}_{p}^{v}\right)^{H}\right] \mathbf{A}_{f, t}^{H}+\mathrm{E}\left[\mathbf{H}^{v}\left(\mathbf{H}^{v}\right)^{H}\right]\right\}\right]
\end{aligned}
$$

where $J=K \times M$. 
Let $\mathrm{i}_{Q}(p)$ and $\mathrm{j}_{Q}(p)$ be the row-index and the column-index, respectively, of an element in a matrix $\mathbf{X}$, having $Q$ rows, that occupies the $p$ th position in $\operatorname{vec}(\mathbf{X})$. We have

$$
\begin{gathered}
\mathrm{i}_{Q}(p)=\operatorname{rem}(p, Q) \\
\mathrm{j}_{Q}(p)=\left\lfloor\frac{p}{Q}\right\rfloor
\end{gathered}
$$

where $\operatorname{rem}(p, Q)$ is the remainder of the division among the integers $p$ and $Q$.

From the definition of $\mathbf{H}^{v}$ and $\hat{\mathbf{H}}_{p}^{v}$ and of the theoretical frequency and time autocorrelation sequences $R_{f}$ and $R_{t}$ [13], we have

$$
\begin{aligned}
& {\left[\mathrm{E}\left[\mathbf{H}^{v}\left(\mathbf{H}^{v}\right)^{H}\right]\right]_{k, l}=R_{f}\left[\mathbf{i}_{K}(k)-\mathbf{i}_{K}(l)\right] R_{t}\left[\mathbf{j}_{K}(k)-\mathbf{j}_{K}(l)\right]} \\
& {\left[\mathrm{E}\left[\mathbf{H}^{v}\left(\hat{\mathbf{H}}_{p}^{v}\right)^{H}\right]\right]_{k, l}=R_{f}\left[\mathbf{i}_{K}(k)-\left(\mathbf{i}_{P}(l) D_{f}-D_{o f f}\left(\mathbf{j}_{P}(l)\right)\right)\right]} \\
& \quad R_{t}\left[\mathbf{j}_{K}(k)-D\left(\mathbf{j}_{P}(l)\right)\right] \\
& {\left[\mathrm{E}\left[\hat{\mathbf{H}}_{p}^{v}\left(\hat{\mathbf{H}}_{p}^{v}\right)^{H}\right]\right]_{k, l}=R_{f}\left[\left(\mathbf{i}_{P}(k)-\mathbf{i}_{P}(l)\right) D_{f}+\right.} \\
& \left.\quad\left(D_{o f f}\left(\mathbf{j}_{P}(k)\right)-D_{o f f}\left(\mathbf{j}_{P}(l)\right)\right)\right] R_{t}\left[D\left(\mathbf{j}_{P}(k)\right)-D\left(\mathbf{j}_{P}(l)\right)\right] \\
& \quad+\sigma_{w}^{2} \delta[k-l] \\
& \mathrm{E}\left[\hat{\mathbf{H}}_{p}^{v}\left(\mathbf{H}^{v}\right)^{H}\right]=\mathrm{E}\left[\mathbf{H}^{v}\left(\hat{\mathbf{H}}_{p}^{v}\right)^{H}\right]^{H}
\end{aligned}
$$

where the function $D(\cdot)$ maps the time index of a column of $\hat{\mathbf{H}}_{p}$ onto the actual time position within the multicarrier frame, and $D_{o f f}(\cdot)$ takes into account the possible offset of the first inserted pilot.

\section{B. Time-Frequency MSE evaluation for channel estimation} algorithms based on channel impulse response estimation

In order to apply (20) to MSE evaluation, a convenient form to express the true coefficients in the frequency domain for the whole frame is given by

$$
\mathbf{H}^{v}=\mathbf{F}_{2} \mathbf{h}^{v}
$$

where vector $\mathbf{h}^{v}$ contains the true time-domain channel impulse response coefficients of all the symbols in a frame, whereas $\mathbf{F}_{2}=\mathbf{I}_{M} \otimes \mathbf{F}_{L}$. From (21), by defining $\boldsymbol{\Theta}=\mathbf{F}_{2} \mathbf{h}^{v}$ and $\hat{\boldsymbol{\Theta}}=\mathbf{B}_{1} \hat{\mathbf{h}}_{p}^{v}$, the MSE on the whole frame can be expressed as

$$
\begin{aligned}
\sigma_{I}^{2} & =\frac{1}{J}\left[\operatorname { t r } \left\{\mathbf{B}_{1} \mathrm{E}\left[\hat{\mathbf{h}}_{p}^{v}\left(\hat{\mathbf{h}}_{p}^{v}\right)^{H}\right] \mathbf{B}_{1}^{H}-\mathbf{B}_{1} \mathrm{E}\left[\hat{\mathbf{h}}_{p}^{v}\left(\mathbf{h}^{v}\right)^{H}\right] \mathbf{F}_{2}^{H}\right.\right. \\
& \left.\left.-\mathbf{F}_{2} \mathrm{E}\left[\mathbf{h}^{v}\left(\hat{\mathbf{h}}_{p}^{v}\right)^{H}\right] \mathbf{B}_{1}^{H}+\mathbf{F}_{2} \mathbf{E}\left[\mathbf{h}^{v}\left(\mathbf{h}^{v}\right)^{H}\right] \mathbf{F}_{2}^{H}\right\}\right]
\end{aligned}
$$

where $J=K \times M$.

In the case of LS/ML estimation we have

$$
\hat{\mathbf{h}}_{p}^{v}=\mathbf{h}_{p}^{v}+\mathbf{S}_{4} \mathbf{w}_{p}^{v}
$$

and equation (26) becomes

$$
\begin{aligned}
& \sigma_{I}^{2}=\frac{1}{J}\left[\operatorname { t r } \left\{\mathbf{B}_{1} \mathrm{E}\left[\mathbf{h}_{p}^{v}\left(\mathbf{h}_{p}^{v}\right)^{H}\right] \mathbf{B}_{1}^{H}-\mathbf{B}_{1} \mathrm{E}\left[\mathbf{h}_{p}^{v}\left(\mathbf{h}^{v}\right)^{H}\right] \mathbf{F}_{2}^{H}\right.\right. \\
& +\mathbf{B}_{1} \mathbf{S}_{4} \mathrm{E}\left[\mathbf{w}_{p}^{v}\left(\mathbf{w}_{p}^{v}\right)^{H}\right] \mathbf{S}_{4}^{H} \mathbf{B}_{1}^{H}-\mathbf{F}_{2} \mathrm{E}\left[\mathbf{h}^{v}\left(\mathbf{h}_{p}^{v}\right)^{H}\right] \mathbf{B}_{1}^{H} \\
& \left.\left.+\mathbf{F}_{2} \mathrm{E}\left[\mathbf{h}^{v}\left(\mathbf{h}^{v}\right)^{H}\right] \mathbf{F}_{2}^{H}\right\}\right]
\end{aligned}
$$

where $\mathbf{w}_{p}^{v}$ is the AWGN vector on the pilot-carrying symbols and $\mathbf{S}_{4}=\mathbf{I}_{|\mathcal{M}|} \otimes \mathbf{S}_{1}$ with $\mathbf{S}_{1}=\left(\mathbf{S}^{H} \mathbf{S}\right)^{-1} \mathbf{S}^{H}, \mathbf{S}=$
$\frac{1}{K} \mathbf{F}^{H} \mathbf{A}_{p} \mathbf{F}_{L}$ and $\mathbf{F}$ the complete Fourier transform matrix [14] [21].

Considering the case of MMSE estimation we have instead

$$
\hat{\mathbf{h}}_{p}^{v}=\mathbf{S}_{5} \mathbf{h}_{p}^{v}+\mathbf{S}_{6} \mathbf{w}_{p}^{v}
$$

and equation (26) becomes

$$
\begin{aligned}
\sigma_{I}^{2}= & \frac{1}{J}\left[\operatorname { t r } \left\{\mathbf{B}_{1} \mathbf{S}_{5} \mathrm{E}\left[\mathbf{h}_{p}^{v}\left(\mathbf{h}_{p}^{v}\right)^{H}\right] \mathbf{S}_{5}^{H} \mathbf{B}_{1}^{H}-\mathbf{B}_{1} \mathbf{S}_{5} \mathrm{E}\left[\mathbf{h}_{p}^{v}\left(\mathbf{h}^{v}\right)^{H}\right] \mathbf{F}_{2}^{H}\right.\right. \\
& +\mathbf{B}_{1} \mathbf{S}_{6} \mathrm{E}\left[\mathbf{w}_{p}^{v}\left(\mathbf{w}_{p}^{v}\right)^{H}\right] \mathbf{S}_{6}^{H} \mathbf{B}_{1}^{H}-\mathbf{F}_{2} \mathrm{E}\left[\mathbf{h}^{v}\left(\mathbf{h}_{p}^{v}\right)^{H}\right] \mathbf{S}_{5}^{H} \mathbf{B}_{1}^{H} \\
& \left.\left.+\mathbf{F}_{2} \mathrm{E}\left[\mathbf{h}^{v}\left(\mathbf{h}^{v}\right)^{H}\right] \mathbf{F}_{2}^{H}\right\}\right]
\end{aligned}
$$

where $\mathbf{S}_{5}=\mathbf{I}_{|\mathcal{M}|} \otimes \mathbf{S}_{2}$ and $\mathbf{S}_{6}=\mathbf{I}_{|\mathcal{M}|} \otimes \mathbf{S}_{3}$, with $\mathbf{V}=\sigma_{w}^{2} \mathbf{C}_{h}^{-1}+\mathbf{S}^{H} \mathbf{S}, \mathbf{S}_{2}=\mathbf{V}^{-1} \mathbf{S}^{H} \mathbf{S}$ and $\mathbf{S}_{3}=\mathbf{V}^{-1} \mathbf{S}^{H}$ [13] [14]. The correlation matrices $\mathrm{E}\left[\mathbf{h}_{p}^{v}\left(\mathbf{h}_{p}^{v}\right)^{H}\right], \mathrm{E}\left[\mathbf{h}_{p}^{v}\left(\mathbf{h}^{v}\right)^{H}\right]$, $\mathrm{E}\left[\mathbf{h}^{v}\left(\mathbf{h}^{v}\right)^{H}\right]$ can be computed under the assumptions of uncorrelated channel path gains with time-varying coefficients, and their expressions is given by

$$
\begin{aligned}
& \mathrm{E}\left[\mathbf{h}^{v}\left(\mathbf{h}^{v}\right)^{H}\right]_{k, l}= P D P\left(\mathrm{i}_{L}(k)\right) \delta\left[\mathrm{i}_{L}(k)-\mathrm{i}_{L}(l)\right] \\
& \times R_{t}\left[\mathrm{j}_{L}(k)-\mathrm{j}_{L}(l)\right] \\
& \mathrm{E}\left[\mathbf{h}_{p}^{v}\left(\mathbf{h}^{v}\right)^{H}\right]_{k, l}= P D P\left(\mathrm{i}_{L}(k)\right) \delta\left[\mathrm{i}_{L}(k)-\mathrm{i}_{L}(l)\right] \\
& \times R_{t}\left[\mathrm{j}_{L}(k)-D\left(\mathrm{j}_{L}(l)\right)\right] \\
& \mathrm{E}\left[\mathbf{h}_{p}^{v}\left(\mathbf{h}_{p}^{v}\right)^{H}\right]_{k, l}= P D P\left(\mathrm{i}_{L}(k)\right) \delta\left[\mathrm{i}_{L}(k)-\mathrm{i}_{L}(l)\right] \\
& \times R_{t}\left[D\left(\mathrm{j}_{L}(k)\right)-D\left(\mathrm{j}_{L}(l)\right)\right] \\
& \mathrm{E}\left[\mathbf{h}^{v}\left(\mathbf{h}_{p}^{v}\right)^{H}\right]=\mathrm{E}\left[\mathbf{h}_{p}^{v}\left(\mathbf{h}^{v}\right)^{H}\right]^{H}
\end{aligned}
$$

where $\left(\mathrm{i}_{L}(k), \mathrm{j}_{L}(k)\right)$ are the frequency-time coordinates of the $k$ th entry of either $\mathbf{h}^{v}$ or $\mathbf{h}_{p}^{v}$, with $L$ the channel length.

\section{Simulation PaRAMETERS}

In order to provide a general term of reference for different communication systems, the LTE-Advanced frame is considered for all the multicarrier communication systems. The LTE downlink communication chain is considered as a basis for all the systems and channel estimation algorithms such as polynomial interpolation, LS, ML and MMSE methods. All the parameters of the simulations are defined according to the LTE specifications for the $3 \mathrm{MHz}$ band: particularly, the LTE frame is composed by ten subframes. Each subframe is formed by two slots (14 symbols) with 15 physical resource blocks (PRB) each, where 120 pilot symbols are inserted on the LTE subframe $(\mathcal{M}=\{0,4,7,11\})$. Moreover, according to the deployment of the LTE pilots, we have set $D_{f}=6$ and $D_{\text {off }}=3$. All the others parameters are reported in Table I. The simulations are performed in a macro-cell vehicular scenario with a multipath fading channel characterized by six Rayleigh distributed paths: in particular we have used the ITU vehicular A channel model, with classical Jake Doppler spectrum in order to take into account the time-variance of the channel [23]. As to the polynomial interpolation algorithms, in the simulation we have considered only linear and spline interpolations which demonstrate the best performance with respect to higher degree polynomials. 


\begin{tabular}{lc}
\hline LTE parameters & Value \\
\hline Transmission BW & $3 \mathrm{MHz}$ \\
Sampling Frequency & $3.84 \mathrm{MHz}$ \\
Subcarrier spacing & $15 \mathrm{kHz}$ \\
FFT size & 256 \\
Number of subcarriers & 180 \\
Symbol Time & $66.67 \mu \mathrm{s}$ \\
CP length & $(4.69 / 18) \times 6$, \\
( $\mu$ s/samples) & $(5.21 / 20) \times 1$ \\
Modulation & QPSK \\
\hline
\end{tabular}

TABLE I

LTE SIMULATION PARAMETERS

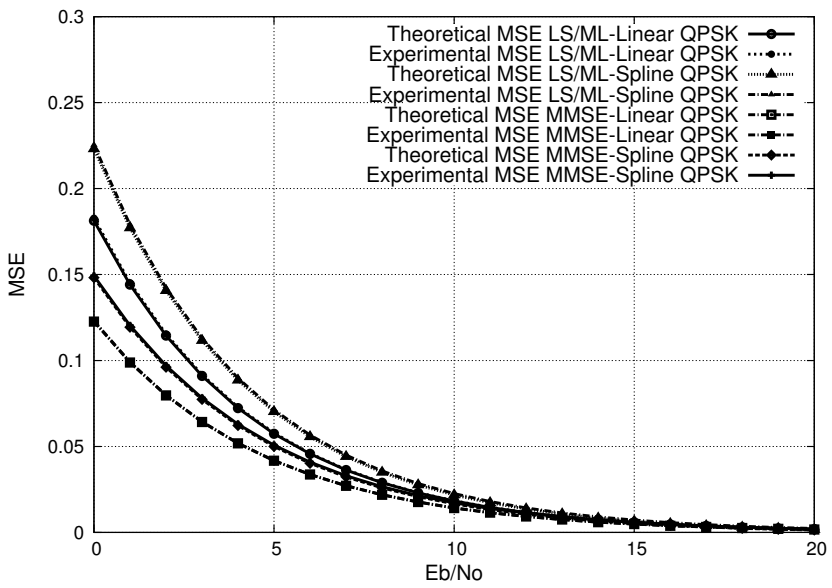

Fig. 1. MSE performance MMSE-Linear and LS/ML-Linear

\section{MSE THEORY VALIDATION AND ANALYSIS}

Firstly, the MSE computation method which has been derived previously is validated by comparing the theoretical values to the ones obtained from simulations in the case of ICI/ISI free systems. In Figure 1, we plot the theoretical MSE curves vs the $E_{b} / N_{0}$ for the algorithms that are based on channel impulse response etimation along with the results of simulations. The methods are referred by indicating the algorithms which have been used for frequency and time estimation. As can be seen, the theoretical and the experimental curves are perfectly overlapped, thus validating the proposed model. Therefore, the theoretical MSE curves provide the ranking of the estimation method: from Figure 1 it is apparent that the MMSE-Linear estimator affords the best performance. For what concerns the validation of the algorithms which are based separable interpolation, the relative results can be found in [18].

\section{Pilots PATTERN DEPLOYMENT DESIGN}

As it is known from [22], the pilots have to be deployed both in a symbol and in consecutive ones: in particular, as depicted in Figure 2, the pilot positions within a symbol have to be equispaced, whereas in the the second case we can design optimal pilot sequences by splitting each pilot sequence of length $P$ into subsequences of length $P / g$ with $g$ number of consecutive symbols, and arbitrarily assigning each subsequence to different symbols. From both channel

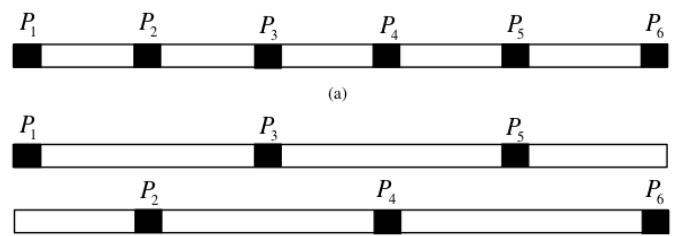

(b)

Fig. 2. (a) Pilot symbols over one OFDM symbol. (b) Pilot symbols over two OFDM symbols

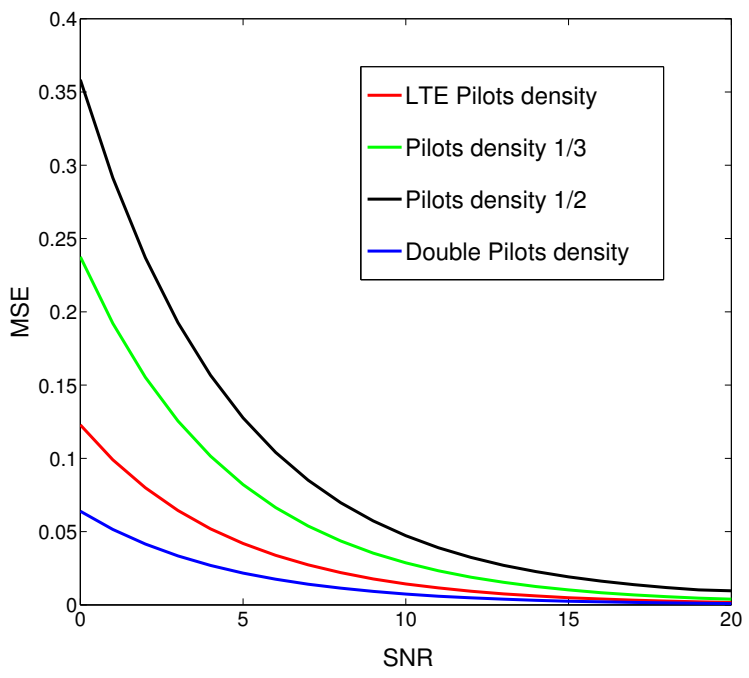

Fig. 3. MSE for variable pilots density

estimation and pilots deployment point of view in GFDM and FBMC frame the same results hold.

In Figure 3 the computed MSE for different pilots configuration is depicted: particularly, the pilots density within the reference LTE downlink subframe has been varied. This result allows to consider communication scenarios where the pilot density has to be varied so adapting the transmission rate to the corresponding traffic as foreseen in the $5 \mathrm{G}$ communications [4], or where the frame dimension has to be changed in order to exploit the white spaces [5] [6].

Thanks to our model the pilot symbol pattern can be defined by resorting to an exaustive research for all the possible value of the pilot pattern parameters [18].

Furthermore, the performance of the whole system depends both on the pilot symbols density and on the SNR: hence, by computing the MSE for different values of the pilot density and the SNR, the families of curves which are described in Figure 4 can be obtained: given an operative target SNR and a target MSE to be achieved, these curves allow to assess the minimum pilot symbols density in order to achieved the requested performance. On the other hand, if a target pilot symbols density and SNR are specified, from Figure 4 the expected MSE can be attained. 


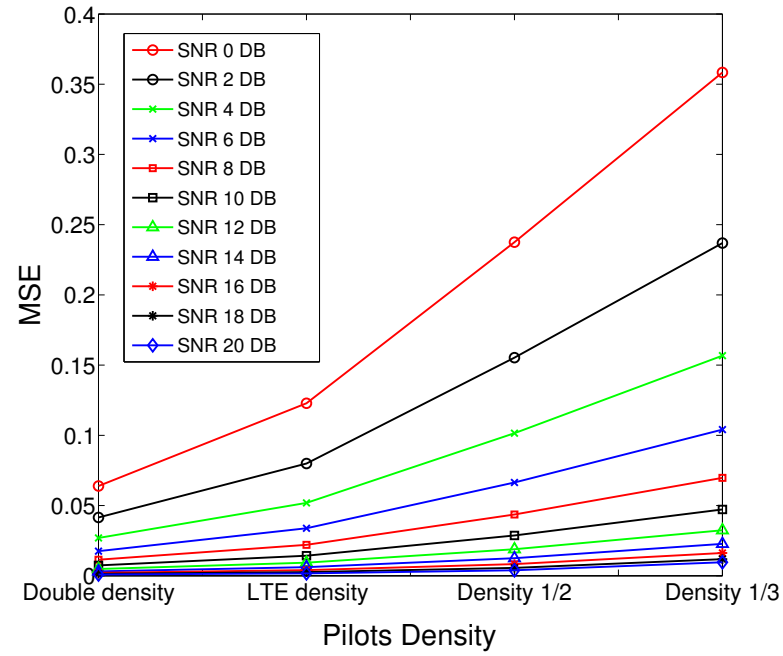

Fig. 4. MSE for different pilots density, normalized to the LTE pilots density

Once obtained the minimmum pilot density we can exploit the indication given in [22] in order to deploy the pilots into the multicarrier frame; afterwards the best pilot pattern and the minimum MSE can be achieved by applying our proposed method. Thus, the proposed method can be seen as a design tool for the selection of the best pilot symbol density and patterns within a general multicarrier frame and particularly, for the $5 \mathrm{G}$ communication systems.

\section{CONCLUSION}

In this paper a general method for the computation of the MSE on the time-frequency domain for pilot based channel estimation techniques in multicarrier system is provided. By generalizing a recently proposed approach, this method permits to define the suitable pilot density in order to afford a target MSE value for a given SNR level. Therefore, in this paper a general design tool for multicarrier systems is proposed which hold also for the future $5 \mathrm{G}$ communication systems.

\section{REFERENCES}

[1] E. Dahlman, S. Parkvall, J. Skold, and P. Beming, $3 G$ Evolution HSPA and LTE for Mobile Broadband. Elsevier Ltd, 2008.

[2] S. Parkvall, A. Furuska, and E. Dahlman, "Evolution of lte toward imt advanced," IEEE Communications Magazine, vol. 49, no. 2, pp. 84-91, 2011.

[3] S. Morosi, S. Jayousi, and E. Del Re, "Cooperative delay diversity in hybrid satellite/terrestrial dvb-sh system," in Proc. of the 2010 IEEE International Conference on Communications, ICC, 2010.

[4] G. Wunder, P. Jung, M. Kasparick, T. Wild, F. Schaich, Y. Chen, S. Brink, I. Gaspar, N. Michailow, A. Festag, L. Mendes, N. Cassiau, D. Ktenas, M. Dryjanski, S. Pietrzyk, B. Eged, P. Vago, and F. Wiedmann, "5gnow: non-orthogonal, asynchronous waveforms for future mobile applications," Communications Magazine, IEEE, vol. 52, no. 2, pp. 97-105, February 2014.

[5] P. Pirinen, "A brief overview of $5 \mathrm{~g}$ research activities," in $5 G$ for Ubiquitous Connectivity (5GU), 2014 1st International Conference on, Nov 2014, pp. 17-22.
[6] N. Michailow, M. Matthe, I. Gaspar, A. Caldevilla, L. Mendes, A. Festag, and G. Fettweis, "Generalized frequency division multiplexing for 5th generation cellular networks," Communications, IEEE Transactions on, vol. 62, no. 9, pp. 3045-3061, Sept 2014.

[7] I. Gaspar, N. Michailow, A. Navarro, E. Ohlmer, S. Krone, and G. Fettweis, "Low complexity gfdm receiver based on sparse frequency domain processing," in Vehicular Technology Conference (VTC Spring), 2013 IEEE 77th, June 2013, pp. 1-6.

[8] B. Farhang-Boroujeny, "Ofdm versus filter bank multicarrier," Signal Processing Magazine, IEEE, vol. 28, no. 3, pp. 92-112, May 2011.

[9] R. Datta, G. Fettweis, Z. Kollar, and P. Horvath, "Fbmc and gfdm interference cancellation schemes for flexible digital radio phy design," in Digital System Design (DSD), 2011 14th Euromicro Conference on, Aug 2011, pp. 335-339.

[10] S. Morosi, T. Bianchi, and F. Gei, "Frequency Domain Multiuser Receivers for an IEEE 802.15.4a Short Range Communication Network," Transactions on Emerging Telecommunications Technologies, vol. 24, no. 6, pp. 545-551, Oct. 2013.

[11] S. Kanchi, S. Sandilya, D. Bhosale, A. Pitkar, and M. Gondhalekar, "Overview of lte-a technology," in Global High Tech Congress on Electronics (GHTCE), 2013 IEEE, Nov 2013, pp. 195-200.

[12] Y. G. Li, L. J. J. Cimini, and N. R. Sollenberger, "Robust channel estimation for ofdm systems with rapid dispersive fading channels," in Proc. of the IEEE International Conference on Communications, ICC 98, 1998.

[13] K. Rajeswari, T. Sangeetha, A. P. Natchammai, M. Nandhini, and S. Thiruvengadam, "Performance analysis of pilot aided channel estimation methods for lte system in time-selective channels," in Industrial and Information Systems (ICIIS), 2010 International Conference on, 2010, pp. $113-118$.

[14] M. Morelli and U. Mengali, "A comparison of pilot-aided channel estimation methods for ofdm systems," Signal Processing, IEEE Transactions on, vol. 49, no. 12, pp. 3065-3073, 2001.

[15] S. Morosi, F. Argenti, M. Biagini, and E. Del Re, "Comparison of channel estimation algorithms for mimo downlink lte systems," in Wireless Communications and Mobile Computing Conference (IWCMC), 2013 9th International, 2013, pp. 953-958.

[16] F. Argenti, M. Biagini, S. Morosi, and E. Del Re, "Time-frequency MSE analysis of linear channel estimation methods for the LTE downlink," Transactions on Emerging Telecommunications Technologies, DOI: 10.1002/ett.2840 in press.

[17] M. Biagini, S. Morosi, F. Argenti, and E. Del Re, "Time-frequency mse analysis for pilot aided channel estimation in ofdm systems," in Proceedings of Wireless Vitae 2014, 2014.

[18] S. Morosi, M. Biagini, F. Argenti, and E. Del Re, "Channel estimation mse analysis for pilot aided ofdm systems," in Proceedings of Euro Med Telco Conference (EMTC), 2014, 2014.

[19] U. Vilaipornsawai and M. Jia, "Scattered-pilot channel estimation for gfdm," in Wireless Communications and Networking Conference (WCNC), 2014 IEEE, April 2014, pp. 1053-1058.

[20] J. Bazzi, P. Weitkemper, and K. Kusume, "Power efficient scattered pilot channel estimation for fbmc/oqam," in SCC 2015; 10th International ITG Conference on Systems, Communications and Coding; Proceedings of, Feb 2015, pp. 1-6.

[21] A. Ancora, C. Bona, and D. Slock, "Down-sampled impulse response least-squares channel estimation for lte ofdma," in Acoustics, Speech and Signal Processing, 2007. ICASSP 2007. IEEE International Conference on, vol. 3, 2007, pp. III-293-III-296.

[22] I. Barhumi, G. Leus, and M. Moonen, "Optimal training design for mimo ofdm systems in mobile wireless channels," Signal Processing, IEEE Transactions on, vol. 51, no. 6, pp. 1615-1624, 2003.

[23] W. Jake, Microwave Mobile Channels. Wiley, 1974. 